\title{
G337.2+0.1: A new X-ray supernova remnant?
}

\author{
J. A. Combi ${ }^{1,2}$, P. Benaglia ${ }^{2,3}$, G. E. Romero ${ }^{2,3}$, and M. Sugizaki ${ }^{4}$ \\ ${ }^{1}$ Departamento de Física (EPS), Universidad de Jaén, Campus Las Lagunillas s/n, 23071 Jaén, Spain \\ e-mail: jcombi@ujaen.es \\ 2 Instituto Argentino de Radioastronomía, C.C.5, (1894) Villa Elisa, Buenos Aires, Argentina \\ 3 Facultad de Ciencias Astronómicas y Geofísicas UNLP, Paseo del Bosque, B1900FWA La Plata, Argentina \\ ${ }^{4}$ Stanford Linear Accelerator Center, 2575 Sand Hill Road, Menlo Park, CA 94025, USA
}

Received 9 December 2004 / Accepted 23 December 2004

\begin{abstract}
We present evidence supporting a SNR origin for the radio source G337.2+0.1, which was discovered along the line of sight to the Norma spiral arm in the MOST $843-\mathrm{MHz}$ radio survey. The radio source is spatially superposed to the unidentified ASCA source AX J1635.9-4719. An analysis of this latter source reveals that its X-ray spectrum, extended nature, and non-variable flux are consistent with what is expected for a SNR. In addition, we have used HI-line observations of the region to look for any effect of the presumed remnant on the ISM. We have found a well-defined minimum centered at the position of the radio source in the velocity range of $\sim-25$ to $-19 \mathrm{~km} \mathrm{~s}^{-1}$. This feature appears as a sharp absorption dip in the spectrum that might be produced when the continuum emission from the SNR candidate is absorbed by foreground gas. Hence we have used it to constrain the distance to the source, which seems to be a young (age $\sim$ a few $10^{3} \mathrm{yr}$ ) and distant $(d \sim 14 \mathrm{kpc})$ SNR. G337.2+0.1 and AX J1635.9-4719 would be the radio/X-ray manifestations of this remnant.
\end{abstract}

Key words. X-ray: individuals: AX J1635.9-4719 - radio continuum: ISM - ISM: supernova remnants - ISM: cosmic rays $\mathrm{X}$-rays: ISM - radiative mechanism: non-thermal

\section{Introduction}

Supernova remnants (SNRs) have long been considered as a primary source of galactic cosmic rays (CRs) with energies up to the knee of the spectrum at $\sim 10^{15.5} \mathrm{eV}$ (e.g. Shklovskii 1953, Ginzburg \& Syrovatskii 1964). First-order Fermi shock acceleration has been suggested as the most likely acceleration mechanism for charged particles in the shells of SNRs (e.g. Bell 1978; Reynolds \& Chevalier 1981). Evidence supporting the presence of $\mathrm{TeV}$ electrons in these objects comes from the detection of synchrotron X-rays in a number of sources such as G347.3-0.5 (Koyama et al. 1997), SN 1006 (1995), Cas A (Allen et al. 1997), and RC W86 (Borkowsky et al. 2001).

A decade ago the detection of SNRs at X-rays was difficult mainly due to the absorption of the soft (i.e., $<3 \mathrm{keV}$ ) X-ray emission by the large column density of gas and dust in the galactic plane. In recent years, with the advent of the new generation of X-ray satellites, the number of galactic SNRs detected in the energy range above $3 \mathrm{keV}$ has been increased considerably. Although the most complete catalog of SNRs is still compiled in the radio band (Green 2004), X-ray instruments such as ASCA, XMM and CHANDRA have provided an important new window to find yet undetected remnants or to confirm candidates originally found at other wavelengths.

In this paper we report the results of a multiwavelength study of the field containing the unidentified X-ray source
AX J1635.9-4719 and the SNR candidate G337.2+0.1. In order to investigate the nature and possible physical connection between both sources, we have performed source crossidentifications with all available astronomical databases and we have re-processed all the relevant data. Our results support the identification of G337.2+0.1 as a new X-ray emitting SNR. In the next section we present a re-analysis of the ASCA data that confirms the extended nature of the source and its non-variable nature. In Sect. 3 we describe the radio continuum and atomic hydrogen observations of the region, and we discuss the origin of the detected sources. We present an overall discussion of the results in Sect. 4 before closing with a summary in Sect. 5.

\section{X-ray analysis of AX J1635.9-4719}

\subsection{Observation and data reduction}

The unidentified X-ray source AX J1635.9-4719 was discovered by the ASCA telescope during a survey of the central region of the galactic plane, performed in the $0.7-10 \mathrm{keV}$ energy range (Sugizaki et al. 2001). The source is located at $(l, b)=$ $\left(337^{\circ} 17,0.06\right),(\alpha, \delta)_{\mathrm{J} 2000.0}=\left(16^{\mathrm{h}} 35^{\mathrm{m}} 56^{\mathrm{s}} 0,-47^{\circ} 19^{\prime} 54^{\prime \prime}\right)$ (1- $\sigma$ uncertainty of $\left.1^{\prime}\right)$. Its integrated flux is $\sim 1.21 \times$ $10^{-12} \mathrm{erg} \mathrm{cm}^{-2} \mathrm{~s}^{-1}$ in the observed X-ray band.

With the aim of studying in more detail the properties of the X-ray source, we have performed a more careful and thorough imaging and spectral analysis of the ASCA data. 


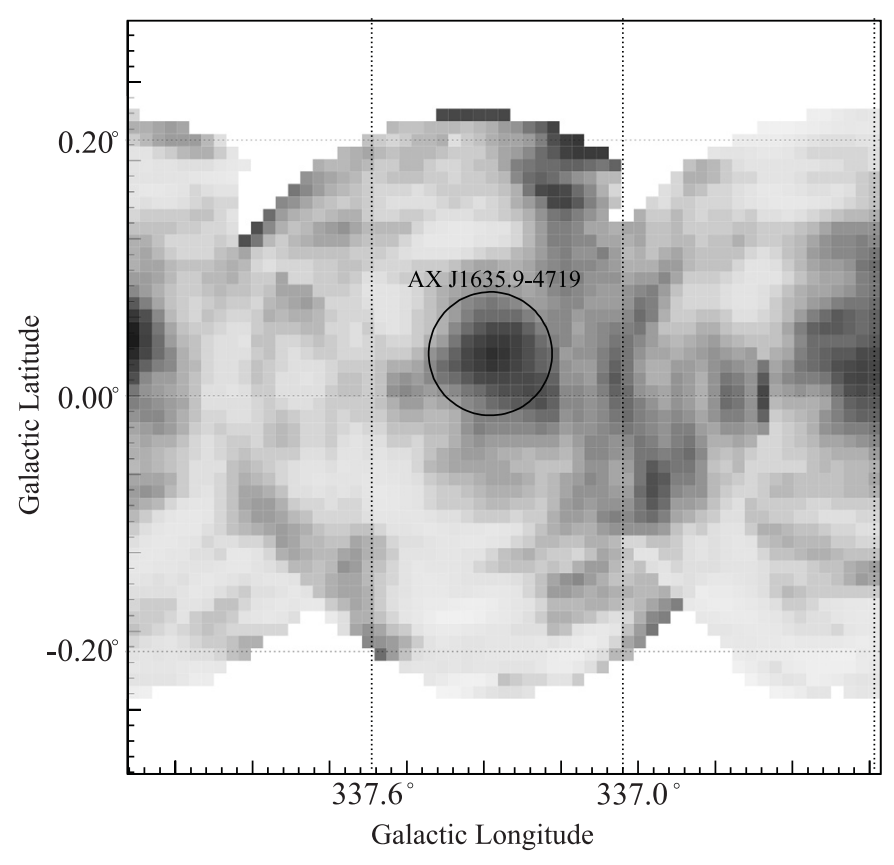

Fig. 1. ASCA GIS mosaic image around AX J1635.9-4719 in the $2-10 \mathrm{keV}$ band. The image is smoothed with a point spread function of the ASCA X-ray telescope with a FWHM of $3^{\prime}$.

The observation of the source was carried out on September 7, 1997 with an exposure of $6.1 \mathrm{ks}$. The data reduction and the extraction of the event for the source were performed following procedures described in Sugizaki et al. (2001). Figure 1 shows the mosaic GIS image of AX J1635.9-4719, where the image is smoothed with the point spread function of the ASCA X-ray telescope with a FWHM of $3^{\prime}$. The image of the source looks like a single peak in the resolution of the instrument. To investigate the spatial extent of the emitting region, we extracted the radial profile of the source in order to compare it with that of the point-spread function of the X-ray telescope. Figure 2 shows the obtained radial profile of the GIS-2 image around AX J1635.9-4719, where the background components of the Galactic and the extra-galactic X-ray emissions are subtracted using the flat-field response. Non-X-ray events were also subtracted following standard procedures. The central core in the inner 2 arcmin is well represented by a point source. However, an extended component clearly exists around the source as it can be seen from the figure.

\subsection{Light curve and spectral analysis}

To examine the X-ray flux variability of AX J1635.9-4719 in more detail, we extracted a light curve and fitted it with a constant model. There is no significant time variation. Coherent periodic pulsation was also investigated by the FFT method: no significant periodic signal can be seen in a period range of $0.5-1000 \mathrm{~s}$. Figure 3 shows the obtained light curve in the whole energy band of $0.7-10 \mathrm{keV}$. The best-fit constant model and the reduced chi-squared $\left(\chi_{v}^{2}\right)$ of the curve are also shown in Fig. 3. The source is non-variable at a confidence above $95 \%$.

We have also re-analyzed the X-ray spectrum of the source. All spectral uncertainties represent $90 \%$ confidence limits

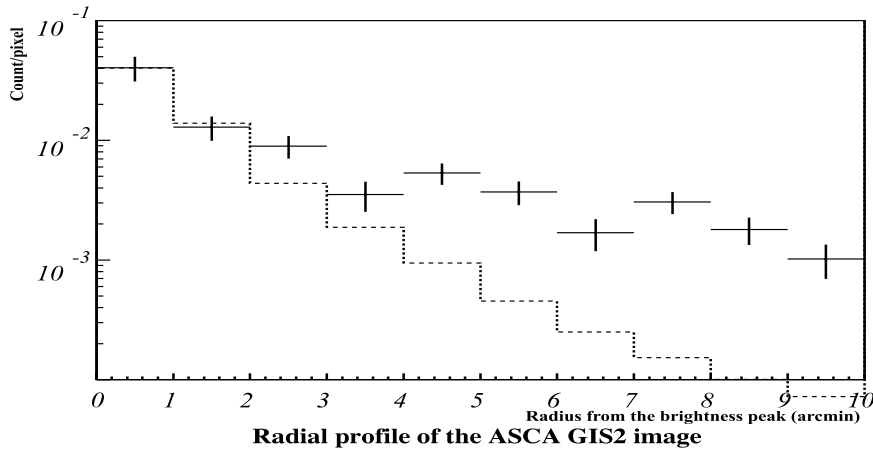

Fig. 2. Radial profile of the X-ray image by GIS-2 around AX J1635.9-4719. The background components of the Galactic and the extra-galactic X-ray emissions are subtracted using the flat-field response. The intrinsic background was also subtracted following standard precedures. Dashed line represents the radial profile expected for a point source.

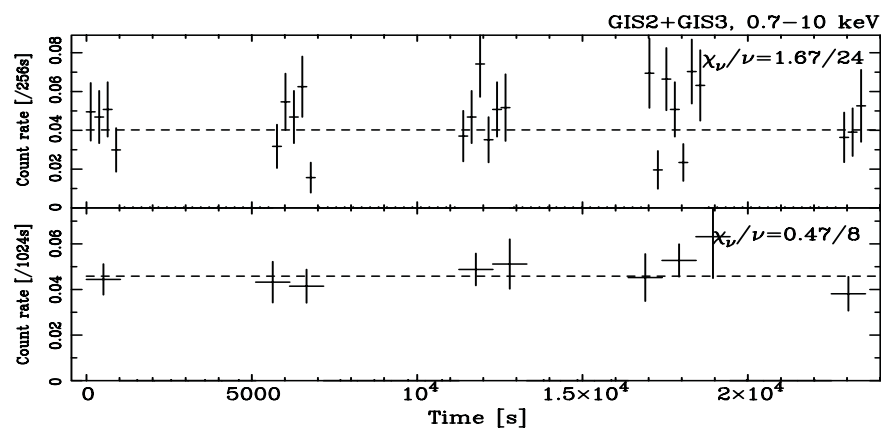

Fig. 3. X-ray light curve of AX J1635.9-4719 during the ASCA observation on Sep. 7, 1997. There is no significant time variation. Coherent periodic pulsation was also investigated by the FFT method. Nothing was found in a period range of $0.5-1000 \mathrm{~s}$.

hereafter. Figure 4 shows the obtained X-ray spectrum in the $0.7-10 \mathrm{keV}$ energy range. The solid line represents the bestfit power-law model with interstellar absorption. The photon index and the absorption column density of the best-fit model are $\Gamma=2.8_{-1.6}^{+2.6}$ and $N_{\mathrm{H}}=15_{-9}^{+15} \times 10^{22} \mathrm{~cm}^{-2}$, respectively. The photon statistics is poor, so it is not possible to provide a well-constrained spectral model. We also attempted to fit thermal emission models of thin-thermal hot plasma coded by Raymond \& Smith (1977) and a blackbody model, and obtained the best-fit model statistically accepted within the $90 \%$ confidence level. Table 1 summarizes these best-fit model parameters. New observations with better statistics are necessary to clearly separate thermal from non-thermal emission in this source. However, it is significant that the absorption column density is of the order of $\sim 10^{23} \mathrm{~cm}^{-2}$.

It is worth noticing that G337.2-0.1 lies well within the $95 \%$ location contour of the unidentified $\gamma$-ray source 3EG J1639-4702 (Hartman et al. 1999). It is located at $(l, b)=$ $\left(337^{\circ} .75,-0.15\right),(\alpha, \delta)_{\mathrm{J} 2000.0}=\left(16^{\mathrm{h}} 39^{\mathrm{m}} 06^{\mathrm{s}},-47^{\circ} 02^{\prime} 28^{\prime \prime}\right)$, and has a radius of about 0.6 . Its $\gamma$-ray flux is $(53.2 \pm 8.7) \times$ $10^{-8} \mathrm{ph} \mathrm{cm}^{-2} \mathrm{~s}^{-1}$, and presents a steep $\gamma$-ray spectral index of $\Gamma=2.5 \pm 0.18$. The EGRET source is non-variable according to both Nolan et al. (2003) and Torres et al. (2001). However, other potential counterparts are within the error box like the 
Table 1. Summary of best-fit parameters of spectral fitting.

\begin{tabular}{lccc}
\hline \hline & \multicolumn{3}{c}{ Model } \\
Parameter & PL & Raymond $^{a}$ & BB \\
\hline$\Gamma$ or $k T^{b}$ & $2.8_{-1.6}^{+2.6}$ & $2.2_{-1.1}^{+13}$ & $1.4_{-0.6}^{+0.9}$ \\
$N_{\mathrm{H}}{ }^{c}$ & $15_{-9}^{+15}$ & $16_{-9}^{+14}$ & $7.7_{-5.7}^{+11}$ \\
$F_{0.7-10 \mathrm{keV}^{d}}$ & 1.2 & 1.2 & 1.2 \\
\hline$\chi_{v}^{2} /$ d.o.f. & $0.91 / 10$ & $0.67 / 10$ & $1.0 / 10$ \\
\hline
\end{tabular}

All errors represent the $90 \%$ confidence limits of statistical uncertainty.

${ }^{a}$ Thin-thermal plasma emission model coded by Raymond \& Smith (1977) with the interstellar absorption.

${ }^{b}$ Power-law photon index or temperature [keV].

${ }^{c}$ Absorption column density $\left[\mathrm{cm}^{-2}\right]$.

${ }^{d}$ Flux in the $0.7-10 \mathrm{keV}$ band $\left[10^{-12} \mathrm{ergs} \mathrm{cm}^{-2} \mathrm{~s}^{-1}\right]$.

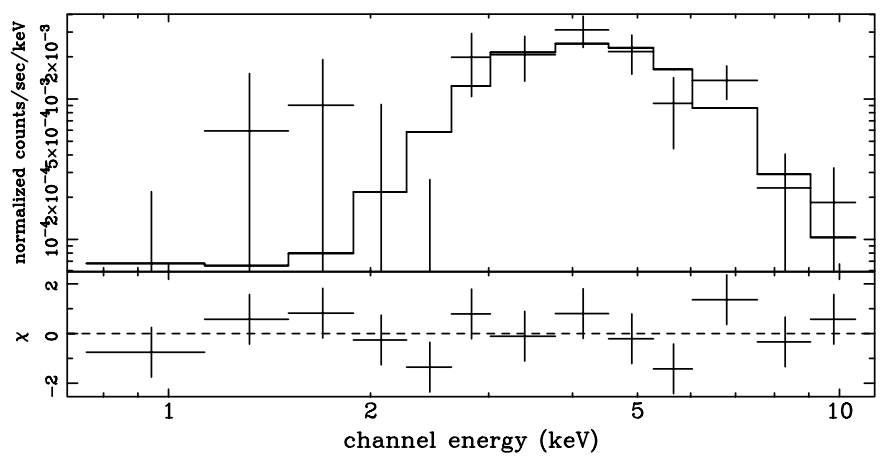

Fig. 4. Energy spectrum observed by ASCA GIS in $0.7-10 \mathrm{keV}$. The solid line represents the best-fit power-law model with interstellar absorption. The photon statistics is very poor, so it is difficult to constrain the spectral model.

microquasar candidate AX J1639.0-4642 (Combi et al. 2004) and the pulsar PSR J1637-4642 (Torres et al. 2003).

\section{Radio continuum and $\mathrm{HI}$ observations towards AX J1635.9-4719}

G337.2+0.1 is a SNR candidate with an angular size of $2^{\prime} \times 3^{\prime}$ discovered in the MOST $843-\mathrm{MHz}$ radio survey (Whiteaok \& Green 1996). The position of the source in galactic and equatorial coordinates is $(l, b)=\left(337^{\circ} .18,+0.06\right)$, $(\alpha, \delta)_{\mathrm{J} 2000.0}=\left(16^{\mathrm{h}} 35^{\mathrm{m}} 55^{\mathrm{s}} 8,-47^{\circ} 19^{\prime} 03^{\prime \prime}\right)$ (3- $\sigma$ uncertainty of $\left.15^{\prime \prime}\right)$. The source has an integrated flux density of $1.6 \pm 0.2 \mathrm{Jy}$ and a mean surface brightness of $2.6 \times 10^{-22} \mathrm{~W} \mathrm{~m}^{-2} \mathrm{~Hz}^{-1} \mathrm{sr}^{-1}$ at $843 \mathrm{MHz}$. In Fig. 5 we show the radio map of G337.2+0.1 at this frequency together with the $2-\sigma$ circle of the best estimated position of AX J1635.9-4719.

In order to find the radio source at higher frequencies we have examined the data from the $4.85 \mathrm{GHz}$ survey (Condon et al. 1993). Applying a filtering process to the data (see Combi et al. 1998 for details of the Gaussian filtering method) we have removed the galactic diffuse emission on scales larger than 8 arcmin and we found a weak and extended radio source coincident with the position of G337.2+0.1. The source has an integrated flux density of $0.39 \pm 0.02 \mathrm{Jy}$. Using the radio flux at

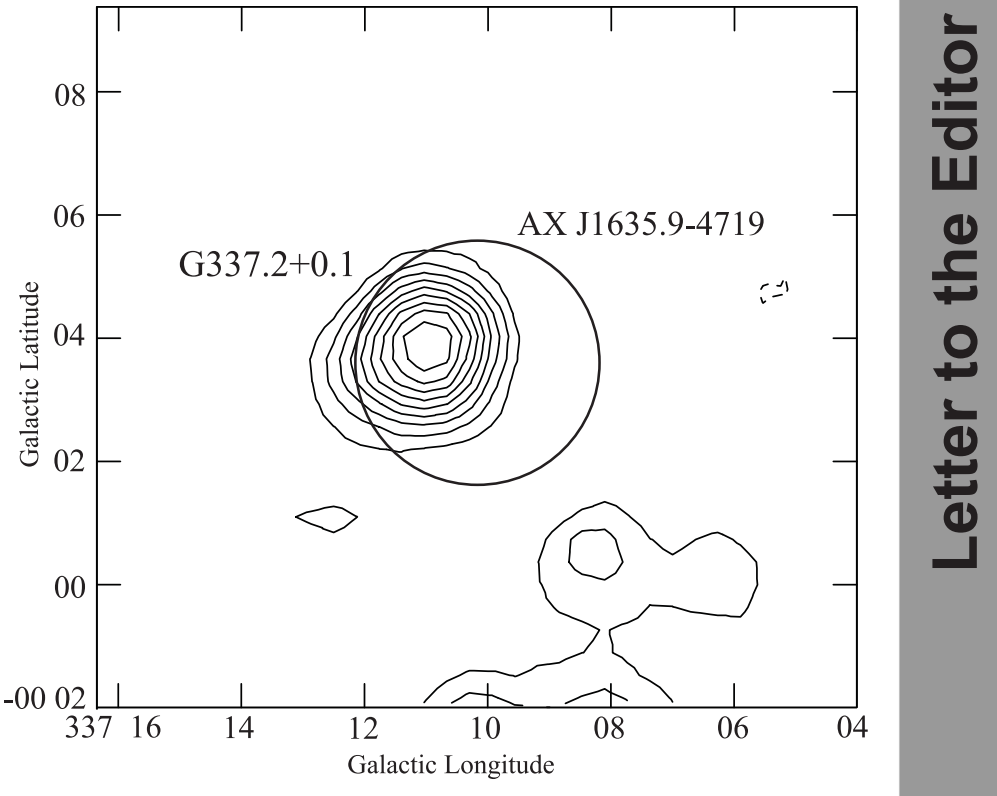

Fig. 5. Countour image of G337.2+0.1 from the MGPS data obtained with MOST at $843 \mathrm{MHz}$. The image size is $12^{\prime} \times 12^{\prime}$. Radio countours are 2, 3, 4, 5, 6, 7 and 8 times the rms noise level of $10 \mathrm{mJy}$. The location of AX J1635.9-4719 is also indicated.

$843 \mathrm{MHz}$ and $4.85 \mathrm{GHz}$ we estimate a mean spectral index for the source of $\sim-0.78$, confirming the non-thermal nature of the radio source.

Since a SN explosion is expected to produce some effect in the ISM, we have extracted a series of HI brightness temperature maps of the region in the velocity interval from $-100 \mathrm{~km} \mathrm{~s}^{-1}$ to $+20 \mathrm{~km} \mathrm{~s}^{-1}$ from the Southern Galactic Plane Survey (SGPS). These data are obtained from a combination of high-resolution interferometric (with the Australia Telescope Compact Array) and low-resolution (with the Parkes Radiotelescope ${ }^{1}$ ) observations (McClure-Griffiths 2001). The survey has angular and velocity resolution of $\sim 2^{\prime}$ and $\sim 0.82 \mathrm{~km} \mathrm{~s}^{-1}$, respectively.

A series of minima can be seen in the HI channel maps, when they are inspected at the highest velocity resolution. Only one of these maps is exactly coincident with the radio continuum source. The HI map integrated over the velocity range from -23 to $-20 \mathrm{~km} \mathrm{~s}^{-1}$ is shown in Fig. 6, where we have superposed the location of the SNR candidate. In the HI spectrum we have found a sharp minimum at $v \sim-20 \mathrm{~km} \mathrm{~s}^{-1}$. Its shape is typical of an absorption feature, hence it can be used to set a lower limit on the distance of the background continuum source. Using the galactic rotation studies of Russeil (2003), we get that the presumed SNR should be at least at $13.5 \mathrm{kpc}$.

\section{Discussion}

Several facts support the identification of the radio source G337.2+0.1 with a SNR. The non-thermal radio spectral index is typical of synchrotron emission from relativistic electrons.

\footnotetext{
1 The ATCA and the Parkes Radiotelescope are part of the Australia Telescope, which is funded by the Commonwealth of Australia for operation as a National Facility managed by CSIRO.
} 


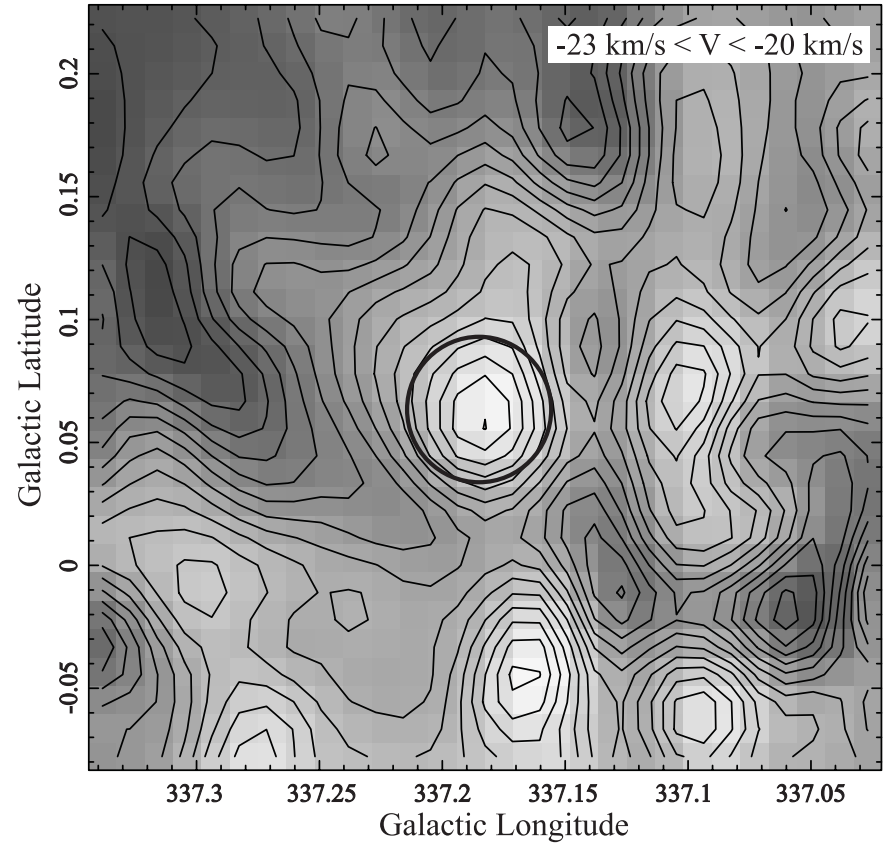

Fig. 6. HI brightness temperature map (contour labels in K) obtained for the velocity range -23 to $-20 \mathrm{~km} \mathrm{~s}^{-1}$. The black circle indicate the SNR position.

At X-rays the flux might also be non-thermal but with a steeper index. This is consistent with the fact that all non-thermal X-ray SNR spectra are substantially curved (e.g. Dyer et al. 2001). The high-energy steepening of the synchrotron spectrum can be due to the effect of losses, the presence of an exponential cutoff in the electron distribution resulting from the failure of the acceleration mechanism at high-energies, and/or non-linear effects. In the case of very young remnants, the particle spectrum can be limited by age (i.e. the remnant could be so young that there was no time to accelerate electrons beyond some maximum energy). However, at the present stage a thermal origin for the X-ray emission cannot be ruled out in this source.

The ASCA data do not allow to specify the morphology of the remnant, although seem to suggest the presence of a central contribution. This might be emission from a pulsar or the result of the low angular resolution and the distance to the source. We have not detected any pulsation in the data.

In addition to the broadband spectrum and the extended, non-variable emission, we have found a sharp absorption feature in the $\mathrm{HI}$ distribution toward the continuum radio source. This can be used to impose a lower bound of $13.5 \mathrm{kpc}$ on the distance to the SNR candidate. If the actual distance is $14 \mathrm{kpc}$, the size of the remnant would be $\sim 6 \mathrm{pc}$, similar to that of SN 1006. Assuming adiabatic expansion in a medium of density $n \sim 0.5 \mathrm{~cm}^{-3}$, the standard Sedov solutions (Sedov 1959) yield an age of $\sim 1500 \mathrm{yr}$ and a shock front velocity of $\sim 215 \mathrm{~km} \mathrm{~s}^{-1}$. At the same distance of $14 \mathrm{kpc}$, the X-ray luminosity of AX J1635.9-4719 is $\sim 4 \times 10^{34} \mathrm{ergs} \mathrm{s}^{-1}$, a quite reasonable value for a young SNR.

\section{Conclusions}

We suggest that G337.2+0.1 is a SNR, being $13.5 \mathrm{kpc}$ a lower limit on its distance, and that AX J1635.9-4719 is the X-ray counterpart of the radio source. G337.2+0.1 might have similar properties to other known galactic SNRs (e.g. SN 1006, G266.2-1.2, G347.3-0.5 and G156.2+5.7) which exhibit a curved broadband synchrotron spectrum. These objects have also high X-ray luminosity, faint radio flux and they are expanding into a low density medium (Allen et al. 2001; Bamba et al. 2001; Koyama et al. 1995, 1997).

Future XMM and Chandra observations will provide a better determination of the spectrum and morphology of this interesting source. The electrons should also cool through inverse Compton scattering off CMB photons (Pohl 1996). This might result in a $\mathrm{TeV}$ gamma-ray source that might be detected with the HESS and CANGAROO III telescopes. Pion decays from interactions of relativistic protons might also contribute in this energy range.

Acknowledgements. We thank Dr. N.M. McClure-Griffiths for providing the HI data and useful discussions, and Dr. K. Koyama for useful comments. J.A.C. is a researcher of the programme Ramón y Cajal funded jointly by the Spanish Ministerio de Ciencia y Tecnología and Universidad de Jaén. He was also supported during this work by CONICET (under grant PEI 6384/03). G.E.R. and P.B. were supported in part by grant BID 1201/OC-AR PICT 03-13291 (ANPCyT) and CONICET.

\section{References}

Allen, G. E., et al. 1997, ApJ, 487, L97

Allen, G. E., Petre, R., \& Gotthelf, E. V. 2001, ApJ, 558, 739

Bamba, A. U., et al. 2001, PASJ, 53, L21

Bell, A. R. 1978, MNRAS, 182, 443

Borkowsky, K. J., et al. 2001, ApJ, 550, 334

Combi, J. A., Romero, G. E., \& Benaglia, P. 1998, A\&A, 333, L91

Combi J. A., et al. 2004, A\&A, 422, 1031

Condon, J. J., Griffith, M. R., \& Wright, A. E. 1993, AJ, 106, 1095

Dyer, K. K., et al. 2001, ApJ, 551, 439

Ginzburg, V. L., \& Syrovatskii, S. I. 1964, The Origin of Cosmic Rays (London: Pergamon Press)

Green, D. A. 2004, A Catalogue of Galactic Supernova Remnants (2004 January version), Mullard Radio Astronomy Observatory, Cavendish Laboratory, Cambridge, UK (available at http://www.mrao.cam.ac.uk/surveys/snrs/)

Hartman, R. C., et al. 1999, ApJS, 123, 79

Koyama, K., et al. 1995, Nature, 378, 255

Koyama, K., et al. 1997, PASJ, 49, L7

McClure-Griffiths, N. M. 2001, Ph.D. Thesis, University of Minnesota Nolan, P. L., et al. 2003, ApJ, 597, 615

Pohl, M. 1996, A\&A, 307, L57

Raymond, J. C., \& Smith, B.W. 1977, ApJS, 35, 419

Reynolds, S. P., \& Chevalier, R. A. 1981, ApJ, 245, 912

Russeil, D. 2003, A\&A, 397, 133

Sedov, L. I. 1959, Similarity and Dimensional Methods in Mechanics (New York: Wiley)

Shklovskii, I. S. 1953, Dokl. Acad. Nauk. SSSR 91, 475

Sugizaki, M., et al. 2001, ApJS, 134, 77

Torres, D. F., et al. 2001, A\&A, 370, 468

Torres, D. F., et al. 2003, Phys. Rep., 382, 303

Whiteoak, J. B. Z., \& Green, A. J. 1996, A\&AS, 118, 329 